\title{
Osh proteins regulate COPII-mediated vesicular transport of ceramide from the endoplasmic reticulum in budding yeast
}

\author{
Kentaro Kajiwara ${ }^{1}$, Atsuko Ikeda ${ }^{1}$, Auxiliadora Aguilera-Romero ${ }^{2}$, Guillaume A. Castillon ${ }^{3}$, Satoshi Kagiwada ${ }^{4}$, \\ Kentaro Hanada ${ }^{5}$, Howard Riezman ${ }^{3}$, Manuel Muñiz ${ }^{2}$ and Kouichi Funato ${ }^{1, *}$
}

\begin{abstract}
Lipids synthesized at the endoplasmic reticulum (ER) are delivered to the Golgi by vesicular and non-vesicular pathways. ER-to-Golgi transport is crucial for maintaining the different membrane lipid composition and identities of organelles. Despite their importance, mechanisms regulating transport remain elusive. Here we report that in yeast coat protein complex II (COPII) vesicle-mediated transport of ceramide from the ER to the Golgi requires oxysterol-binding protein homologs, Osh proteins, which have been implicated in lipid homeostasis. Because Osh proteins are not required to transport proteins to the Golgi, these results indicate a specific requirement for the Osh proteins in the transport of ceramide. In addition, we provide evidence that Osh proteins play a negative role in COPII vesicle biogenesis. Together, our data suggest that ceramide transport and sphingolipid levels between the ER and Golgi are maintained by two distinct functions of Osh proteins, which negatively regulate COPII vesicle formation and positively control a later stage, presumably fusion of ceramide-enriched vesicles with Golgi compartments.
\end{abstract}

KEY WORDS: Oxysterol-binding protein, Ceramide, COPII, Vesicular transport, Yeast

\section{INTRODUCTION}

Trafficking of newly synthesized lipids and proteins from the ER is crucial and must be tightly regulated to maintain the specific membrane composition and function of each organelle. Coat protein complex II (COPII)-coated vesicles mediate transport of both lipids and proteins to the Golgi compartment (Gillon et al., 2012; Perry and Ridgway, 2005). In addition to COPII-dependent vesicular transport, non-vesicular mechanisms are used for delivery of lipids from the ER to other compartments (Lev, 2012; Perry and Ridgway, 2005; Prinz, 2010). The bestcharacterized example of a lipid that is delivered to the Golgi by non-vesicular mechanisms is ceramide (Perry and Ridgway, 2005; Prinz, 2010). It is converted in the Golgi to more complex sphingolipids and is involved in the formation of sphingolipidand sterol-enriched domains that are proposed to be involved in protein sorting and signaling (van Meer et al., 2008). In yeast, the

${ }^{1}$ Department of Biofunctional Science and Technology, Graduate School of Biosphere Science, Hiroshima University, Hiroshima 739-8528, Japan.

${ }^{2}$ Department of Cell Biology, University of Seville, 41012 Seville, Spain. ${ }^{3}$ NCCR Chemical Biology, Department of Biochemistry, Sciences II, University of Geneva, $\mathrm{CH}-1211$ Geneva 4, Switzerland. ${ }^{4}$ Department of Biological Science, Nara Women's University, Nara 630-8506, Japan. ${ }^{5}$ Department of Biochemistry and Cell Biology, National Institute of Infectious Diseases, Tokyo 162-8640, Japan.

*Author for correspondence (kfunato@hiroshima-u.ac.jp) molecular mechanisms of COPII vesicle formation have been extensively studied (Miller and Barlowe, 2010; Sato and Nakano, 2007) and our previous study showed that ceramides synthesized de novo in the ER are transported to the Golgi by both COPIIdependent vesicular transport and COPII-independent nonvesicular transport (Funato and Riezman, 2001). Additionally the COPII-independent pathway requires membrane contacts between the ER and Golgi but not ATP (Funato and Riezman, 2001). To date the regulatory mechanisms of the COPIIindependent pathway remain largely unknown.

Oxysterol-binding protein (OSBP) and OSBP-related proteins (ORPs) make up a conserved family of lipid-binding proteins that are implicated in sterol homeostasis, phospholipid metabolism, vesicular transport and regulation of non-vesicular ceramide transport mediated by ceramide transport protein (CERT) (Beh et al., 2012; Beh and Rine, 2004; de Saint-Jean et al., 2011; Fang et al., 1996; Georgiev et al., 2011; Johansen et al., 2012; Perry and Ridgway, 2006; Schulz et al., 2009; Stefan et al., 2011; Vihervaara et al., 2011). Budding yeast lacks a CERT homolog, but expresses ORPs. Osh4p, one of seven yeast ORPs (Osh1p-Osh7p) that share overlapping essential functions, has binding sites for sterol and phosphatidylinositol 4-phosphate (PtdIns4P) (de Saint-Jean et al., 2011; Im et al., 2005). Moreover, it has been experimentally demonstrated that Osh4p is involved in sterol transport between membranes (de Saint-Jean et al., 2011). A sterol-PtdIns4P exchange and control of their levels might be coupled to the regulatory roles of Osh $4 p$ in post-Golgi vesicular transport involving Sec14p and in polarized exocytosis (Beh et al., 2012; Johansen et al., 2012). However, a recent study argued against a role of Osh4p in sterol transfer (Beh et al., 2012; Georgiev et al., 2011). Therefore, the exact function of Osh proteins is not yet clear but they might be lipid sensors that control lipid and protein metabolism (Stefan et al., 2011; Vihervaara et al., 2011) or regulators of membrane contact site formation (Schulz et al., 2009) or membrane composition and organization (Georgiev et al., 2011).

In this study, we show that COPII-dependent vesicular transport of ceramide but not proteins from the ER to the Golgi requires Osh proteins. We also report that, in addition to a function as positive regulators, Osh proteins act as negative regulators of COPII vesicle biogenesis. These findings show that vesicular ceramide transport can be dissociated from protein transport suggesting either separate vesicles or specific sorting mechanisms. Thus, sphingolipid levels in cellular membranes could be regulated by ceramide vesicular transport controlled by Osh proteins.

\section{RESULTS}

Osh proteins are needed for efficient ER to Golgi ceramide transport To further understand the functions of the yeast Osh proteins in lipid homeostasis, we analyzed the synthesis of complex sphingolipids in a mutant strain (osh $\Delta$ osh4-1) that has a 
A
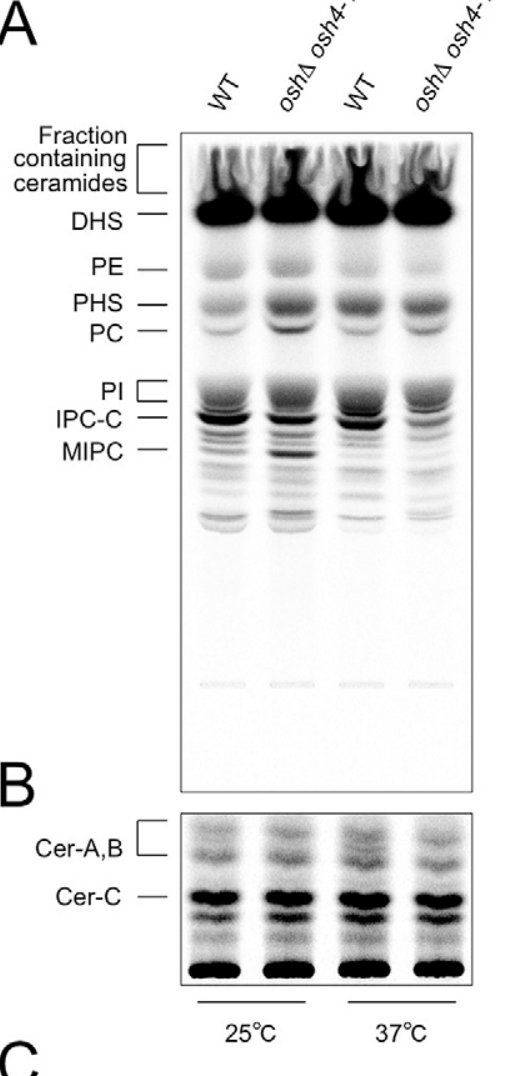

C

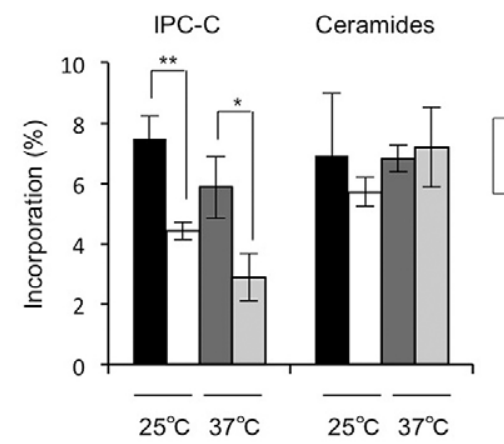

$\mathrm{H}$

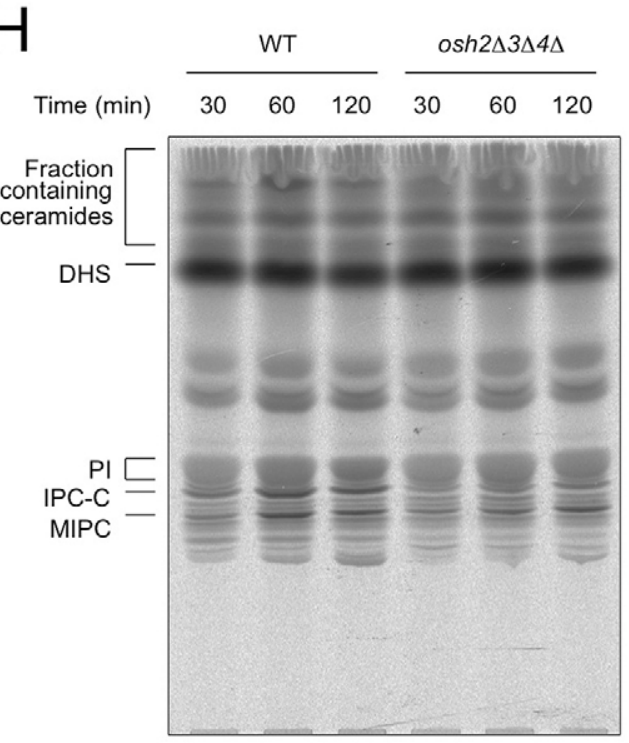

E
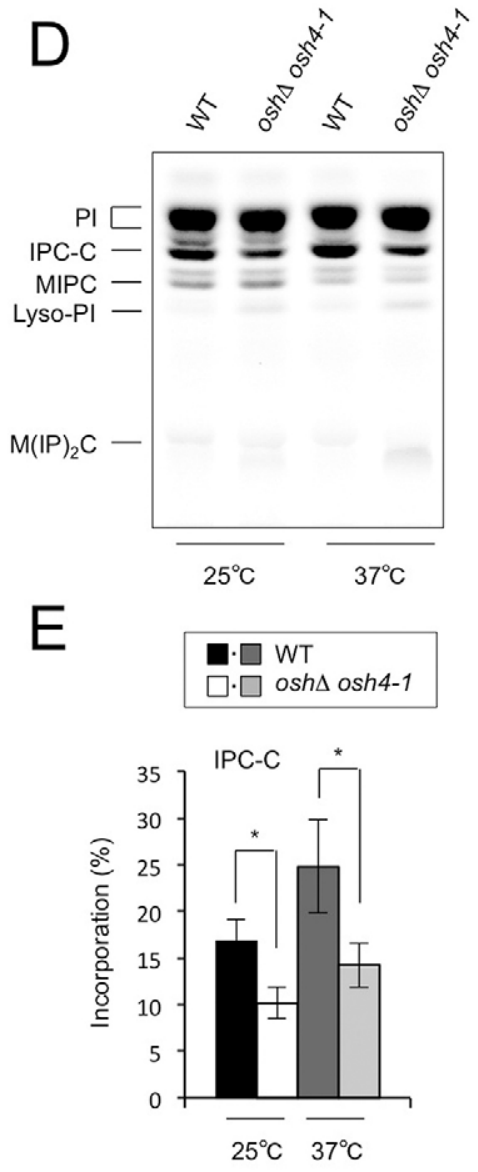

G
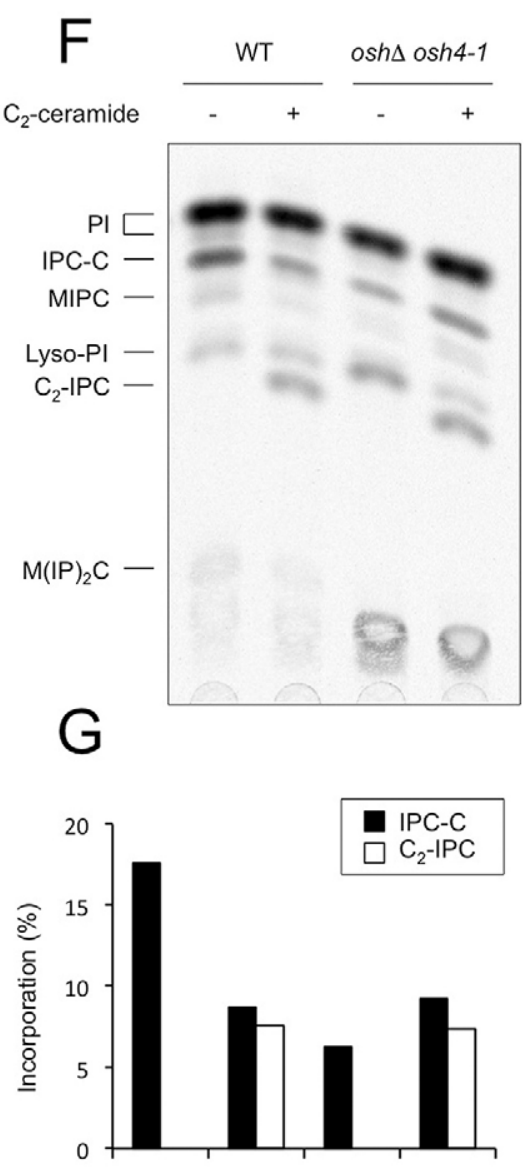

$\mathrm{C}_{2}$-ceramide
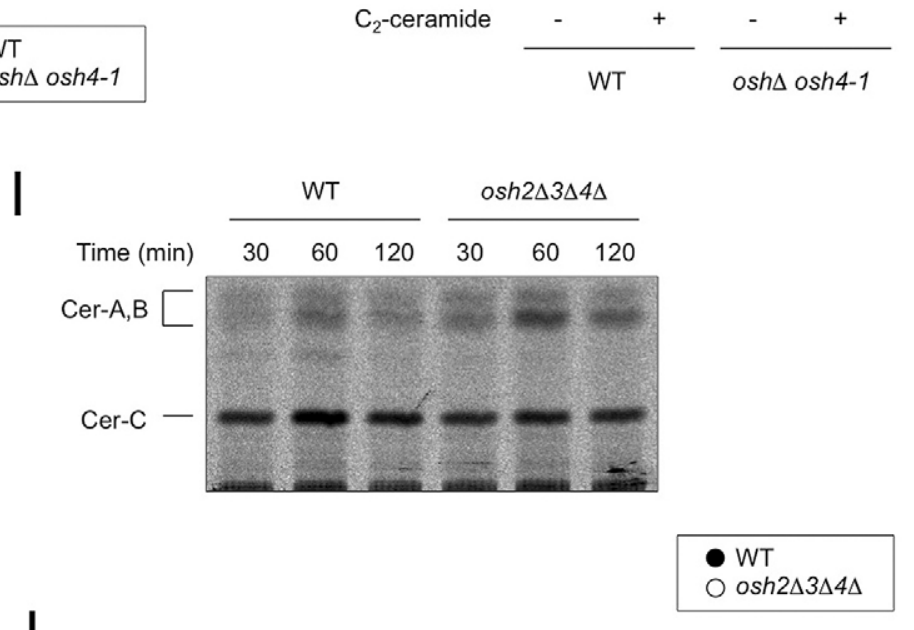

$J$

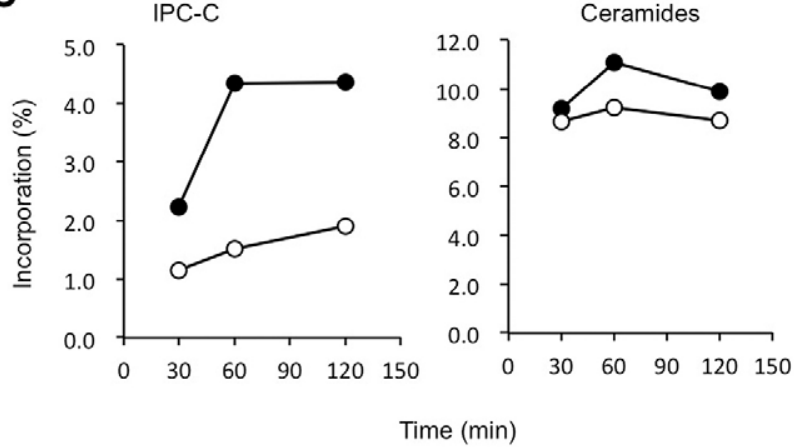

Fig. 1. See next page for legend. 
Fig. 1. Osh proteins are required for efficient transport of ceramide from the ER to the Golgi. (A-E) Cells were grown at either $25^{\circ} \mathrm{C}$ or shifted to $37^{\circ} \mathrm{C}$ for 20 minutes and labeled with $\left[{ }^{3} \mathrm{H}\right] \mathrm{DHS}(\mathrm{A}-\mathrm{C})$ or $\left[{ }^{3} \mathrm{H}\right]$ myo-inositol $(D, E)$ at the same temperature for 60 minutes. The labeled lipids were extracted and applied to TLC plates that were developed with solvent system II (A) or system I (D). Fractions containing ceramides in A, were collected by scraping and the lipid extracts from the silica were analyzed by TLC using solvent system III (B). Incorporation of $\left[{ }^{3} \mathrm{H}\right] \mathrm{DHS}$ into IPC-C or ceramides (Cer-A, -B and -C; C) and of $\left[{ }^{3} \mathrm{H}\right]$ myo -inositol into IPC-C (E) was quantified. Incorporation was determined as a percentage of the total radioactivity. Data are means \pm s.d. $(n=3)$ of three independent experiments. ${ }^{*} P<0.05$; ${ }^{* *} P<0.01$; compared with WT. $(F, G)$ Cells were preincubated at $37^{\circ} \mathrm{C}$ for 20 minutes and labeled with $\left[{ }^{3} \mathrm{H}\right]$ myo-inositol at $37^{\circ} \mathrm{C}$ for 60 minutes in the presence or absence of $\mathrm{C}_{2}$-ceramide. The labeled lipids were extracted and analyzed by TLC using solvent system I (F). The incorporation into IPC-C and $\mathrm{C}_{2}$-IPC was determined as a percentage of the total radioactivity $(G)$. $(\mathrm{H}-\mathrm{J})$ Cells were labeled with $\left[{ }^{3} \mathrm{H}\right] \mathrm{DHS}$ at $25^{\circ} \mathrm{C}$ for the indicated times. The labeled lipids were extracted and analyzed by TLC using solvent system II $(\mathrm{H})$. Fractions containing ceramides in $\mathrm{H}$ were collected and the lipid extracts were analyzed by TLC using solvent system III (I). The incorporation (\%) into IPC-C and ceramides were determined (J). PE, phosphatidylethanolamine; PHS, phytosphingosine; PC, phosphatidylcholine; PI, phosphatidylinositol; IPC-C, inositolphosphorylceramide subclasses C; MIPC, mannosyl inositolphosphorylceramide; Cer-A, -B and -C, different ceramide species, which were identified using mutants that are defective in the biosynthesis of specific ceramide species and by chemical treatment as described previously (Funato and Riezman, 2001; Haak et al., 1997); Lyso-PI, lysophosphatidylinositol; $\mathrm{M}(\mathrm{IP})_{2} \mathrm{C}$, mannosyl di(inositolphosphoryl)ceramide.

temperature-sensitive osh4-1 allele of the OSH4 gene and deletions of the other six OSH genes (Beh and Rine, 2004). In this osh $\Delta$ osh4-1 mutant strain at both permissive and nonpermissive temperatures, incorporation of $\left[{ }^{3} \mathrm{H}\right]$ dihydrosphingosine (DHS) into inositol phosphorylceramide subclass C (IPC-C), one of the yeast complex sphingolipids, was reduced to half that of the wild-type strain (Fig. 1A,C), despite the fact that the two strains synthesized similar amounts of ceramides (Fig. 1B,C), which are the direct precursors of IPCs. In osh $\Delta$ osh $4-1$, we also observed a dramatically reduced incorporation of $\left[{ }^{3} \mathrm{H}\right] m y o$-inositol into IPC-C (Fig. 1D,E). The IPC synthesis defects observed in the osh $\Delta$ osh4-1 strain could be explained by either slower transport kinetics of ceramide from the ER to the Golgi or a reduction in the activity of IPC synthase. To discriminate between these two possibilities we measured the IPC synthase activity in vivo as described previously (Kajiwara et al., 2008). In this assay $\mathrm{C}_{2^{-}}$ ceramide added exogenously to cells reaches the Golgi apparatus through a diffusion-mediated or an endocytic route. There the IPC synthase, Aurlp, modifies $\mathrm{C}_{2}$-ceramide into $\mathrm{C}_{2}$-IPC. We did not find any significant difference in synthesis of $\mathrm{C}_{2}$-IPC in wild-type and osh $\Delta$ osh $4-1$ cells (Fig. 1F,G), which suggested that osh $\Delta$ osh $4-1$ cells are not compromised for IPC synthase activity. Thus, we conclude that the IPC synthesis defect of osh $\Delta$ osh $4-1$ cells results from a deficiency in ceramide transport.

Because Osh proteins are likely to play a role in transport of ceramides from the ER to the Golgi, we wanted to determine whether one of the Osh proteins or a specific subset is involved in the ceramide transport. When mutants lacking one of the Osh proteins were labeled with $\left[{ }^{3} \mathrm{H}\right] \mathrm{DHS}$, no significant difference was found in the incorporation into IPC-C (supplementary material Fig. S1A). Because mutant strains that have abnormal sphingolipid metabolism exhibit altered sensitivity to inhibitors of sphingolipid synthesis, such as aureobasidin A (AbA) (Epstein et al., 2012; Kajiwara et al., 2012; Kajiwara et al., 2008; Malathi et al., 2004), we measured growth of osh $\Delta$ deletion strains on plates containing either $\mathrm{AbA}$, which targets IPC synthase or myriocin (Myr), which targets serine palmitoyltransferase (Horvath et al., 1994; Nagiec et al., 1997). As reported previously, the osh3 $\Delta$ strain was resistant to Myr (Yano et al., 2004), whereas it was hypersensitive to AbA (supplementary material Fig. S1B). Interestingly, osh $2 \Delta$ and osh $4 \Delta$ strains showed resistance to $\mathrm{AbA}$. AbA and Myr had no or minimal effects on the fitness of other deletion strains relative to WT. The $\mathrm{AbA}$ resistance of $o s h 2 \Delta$ and $o s h 4 \Delta$ strains was suppressed by the additional deletion of $\mathrm{OSH} 3$ (supplementary material Fig. S1B) and $o s h 2 \triangle \mathrm{AbA}$ resistance was rescued by overexpression of Osh4p (data not shown). These results suggest that Osh2p and Osh $4 p$ act in a same pathway and Osh3p act antagonistically to the Osh2p/Osh4p-dependent pathway, providing a link between Osh $2 p / O s h 4 p$ and Osh $3 p$ function to maintain sphingolipid homeostasis or to sense changes in sphingolipid levels.

The findings led us to assess the incorporation of $\left[{ }^{3} \mathrm{H}\right] \mathrm{DHS}$ into IPC in the double and triple mutants (supplementary material Fig. S1A). Although no reductions were observed in the double mutants, the osh $2 \Delta$ osh $3 \Delta$ osh $4 \Delta$ triple mutant showed a substantial decrease in IPC-C synthesis. Similar results were obtained when cells were labeled with $\left[{ }^{3} \mathrm{H}\right]$ myo-inositol (data not shown). We also found that the triple mutant synthesizes IPC-C at rates markedly slower than the wild-type (Fig. 1H,J), despite equal levels of ceramides (Fig. 1I,J), suggesting that Osh2p, Osh $3 p$ and Osh4p are required for the efficient transport of ceramide from the ER to the Golgi. Thus, it is likely that whereas Osh $2 p / O s h 4 p$ and Osh $3 p$ have distinct functions, presumably in sensing changes in sphingolipid levels, they have overlapping functions in maintaining IPC synthesis. The osh2 $\Delta$ osh $3 \Delta \operatorname{osh} 4 \Delta$ triple mutant had modest effects on mannosyl IPC (MIPC) synthesis (Fig. 1H). This may be due to an increased activity in converting IPC to MIPC, which might explain the enhanced incorporation into MIPC that was observed in the osh $\Delta$ osh4-1 mutant at $25^{\circ} \mathrm{C}$ (Fig. 1A). In addition, a steady-state metabolic labeling with $\left[{ }^{3} \mathrm{H}\right]$ myo-inositol by a 1 -hour pulse and a 20 -hour chase showed a decreased incorporation into complex sphingolipids in the triple mutant (data not shown), consistent with an increased sensitivity to both $\mathrm{AbA}$ and $\mathrm{Myr}$ (supplementary material Fig. S1B).

\section{Osh proteins are needed for vesicular transport of ceramides to the Golgi compartment}

In the budding yeast Saccharomyces cerevisiae, ceramide is transported to the Golgi by both vesicular and non-vesicular transport pathways (Funato and Riezman, 2001). Although the mechanisms that regulate non-vesicular transport in yeast are largely unknown, it was suggested that non-vesicular transport occurs by an ATP-independent mechanism, through membrane contact sites between the ER and Golgi (Funato and Riezman, 2001). Because Osh proteins appear to function at membrane contact sites (Schulz et al., 2009; Stefan et al., 2011; Beh et al., 2012), it is possible that Osh proteins might be needed for nonvesicular transport of ceramide. To investigate this possibility, we measured the incorporation of $\left[{ }^{3} \mathrm{H}\right]$ myo-inositol into IPC-C in the osh $2 \Delta \operatorname{osh} 3 \Delta$ osh $4 \Delta$ strain under conditions that block vesicular transport. If indeed Osh proteins regulate non-vesicular transport, but not vesicular transport of ceramide, then the $\operatorname{ssh} 2 \Delta \operatorname{osh} 3 \Delta$ osh4A mutant should show an additional reduction of IPC synthesis by blocking vesicular trafficking. Unexpectedly, IPC synthesis in the $\operatorname{osh} 2 \Delta \operatorname{osh} 3 \Delta \operatorname{osh} 4 \Delta$ mutant, in comparison with the wild type, was hardly affected by ATP depletion using $\mathrm{NaN}_{3}$ and $\mathrm{NaF}$ (Fig. 2A,B). This suggests that the supply of ceramide 


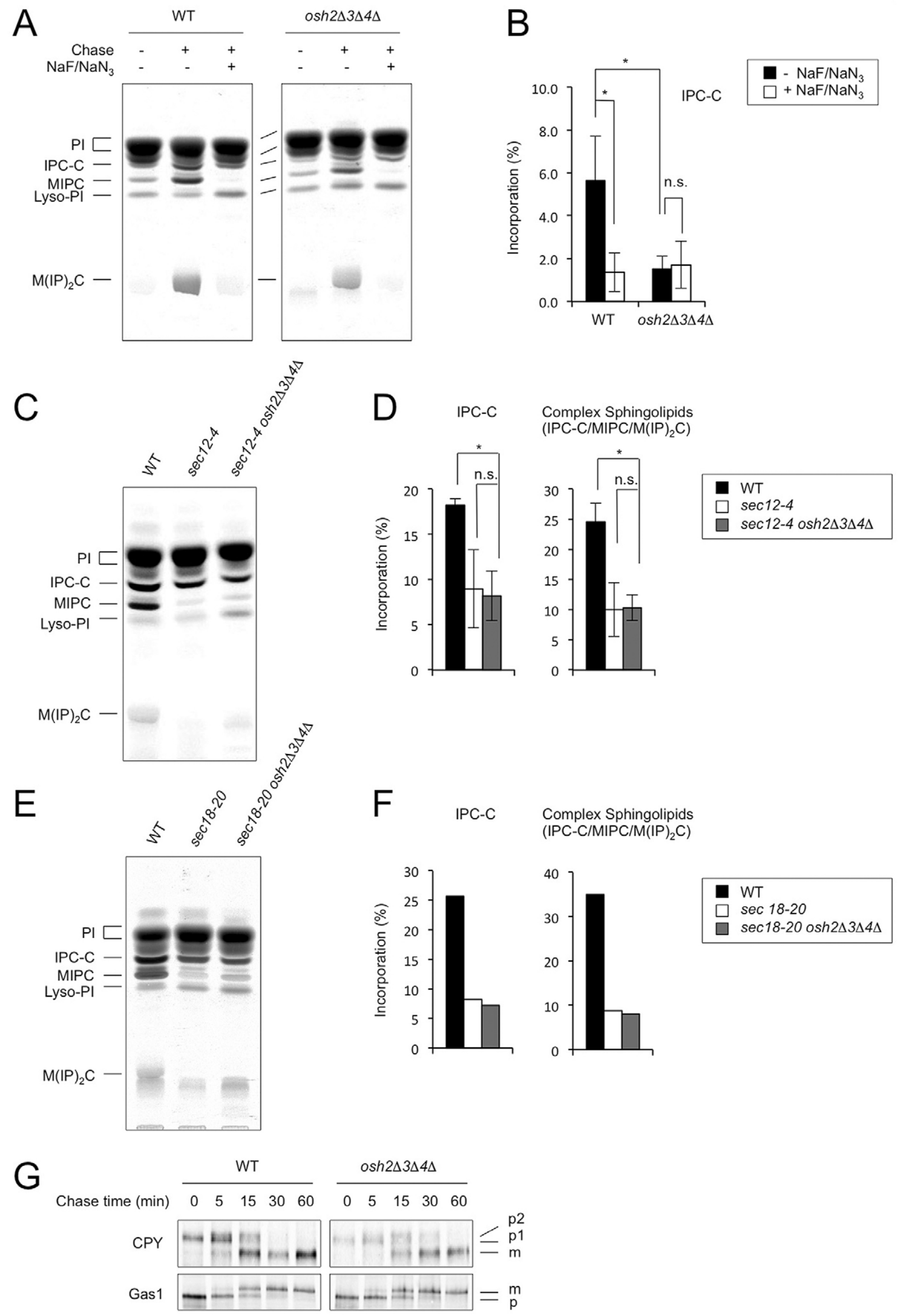

Fig. 2. See next page for legend. 
Fig. 2. Osh proteins have a cargo-specific function in vesicular transport from the ER. (A,B) Cells labeled with $\left[{ }^{3} \mathrm{H}\right]$ myo-inositol at $4{ }^{\circ} \mathrm{C}$ for 60 minutes were subjected to ATP depletion ( $\mathrm{NaF}$ and $\mathrm{NaN}_{3}$ treatment) and then chased at $25^{\circ} \mathrm{C}$ for 60 minutes. The labeled lipids were extracted and analyzed by TLC using solvent system I (A). The incorporation into IPC-C was determined as a percentage of the total radioactivity minus the incorporation (\%) in non-chased cells (B). (C-F) Cells grown at $25^{\circ} \mathrm{C}$ were shifted to $37^{\circ} \mathrm{C}$ for 20 minutes and labeled with $\left[{ }^{3} \mathrm{H}\right]$ myo-inositol at $37^{\circ} \mathrm{C}$ for 60 minutes. The labeled lipids were extracted and analyzed by TLC using solvent system I $(C, E)$. The incorporation (\%) into IPC-C and into total complex sphingolipids [IPC-C, MIPC and $M(I P)_{2} C$ ] were determined $(D, F)$. Data in $\mathrm{B}$ and $\mathrm{D}$ are means \pm s.d. $(n=3)$ of three independent experiments. ${ }^{*} P<0.05$; n.s., not significant. (G) Cells were labeled with [ ${ }^{35}$ S]methionine for 5 minutes and chased at $25^{\circ} \mathrm{C}$ for the indicated times. Proteins were immunoprecipitated, resolved by SDS-PAGE, and analyzed with a phosphoimager. p1 and p2, ER and Golgi forms of CPY, respectively; $p, E R$ form of Gas1; m, mature form of CPY or Gas1.

for the remaining IPC synthesis in the osh triple mutant is brought by non-vesicular transport, which is unaffected by ATP depletion; therefore revealing that Osh proteins are involved in vesicular transport of ceramide. Consistent with this, a triple deletion of $\mathrm{OSH} 2, \mathrm{OSH} 3$ and $\mathrm{OSH} 4$ had no additive effect on the rates of IPC or complex sphingolipid synthesis at non-permissive temperature in sec12-4 (Fig. 2C,D) and sec18-20 (Fig. 2E,F) mutants, which have defective vesicular transport (Funato and Riezman, 2001).

In addition, we found that CERT, which mediates nonvesicular transfer of ceramide in mammalian cells (Hanada et al., 2003), failed to complement the defects of both incorporation into IPC-C in the osh $2 \Delta$ osh $3 \Delta$ osh $4 \Delta$ cells (supplementary material Fig. S2A,B) and growth at nonpermissive temperature in osh $\Delta$ osh $4-1$ cells (supplementary material Fig. S2C), although it could be due to differential substrate specificities of Osh proteins and CERT in ceramide transfer. Osh $4 p$ also failed to complement the defect of sphingomyelin synthesis in a Chinese hamster ovary (CHO) cell line LY-A mutant (supplementary material Fig. S2D), which has a missense mutation in the CERT (Hanada et al., 2003).

\section{Osh proteins contribute in a SAC1-independent manner to IPC synthesis}

Osh proteins stimulate Saclp phosphoinositide phosphatase activity (Stefan et al., 2011). Because deletion of SAC1 reduces complex sphingolipid levels (Brice et al., 2009), Osh proteinmediated ceramide transport may be driven by a Sac1p-dependent process. We found, however, that overexpression of Saclp rescued the IPC synthesis defect and PtdIns $4 P$ accumulation as observed in sac1 $\Delta$ cells (supplementary material Fig. S3A), but not the IPC synthesis defect in osh $2 \Delta$ osh $3 \Delta$ osh $4 \Delta$ cells (supplementary material Fig. S3B), suggesting that Osh proteins contribute in a $S A C 1$-independent manner to IPC synthesis.

\section{Osh proteins are not required for vesicular transport of proteins to the Golgi compartment}

If Osh proteins are needed for efficient vesicular transport of ceramide from the ER, then we predict that deletion of OSH genes should affect the ER-to-Golgi transport of glycosylphosphatidylinositol-anchored proteins (GPI-APs), because we previously proposed that ceramides and GPI-APs are co-transported in the same vesicles from the ER in yeast (Kajiwara et al., 2008). However, the triple deletion of $\mathrm{OSH}$, $\mathrm{OSH} 3$ and $\mathrm{OSH} 4$ had no effect on the maturation of the GPI-AP, Gas1p, consistent with normal transport to the Golgi (Fig. 2G).
Biogenesis of a non-GPI-AP, carboxypeptidase Y (CPY), was also unaffected in osh $2 \Delta$ osh $3 \Delta$ osh $4 \Delta$ cells (Fig. 2G). These results are consistent with a previous study that used strains lacking all $\mathrm{OSH}$ (Beh and Rine, 2004), and provide further evidence that Osh proteins are not required for vesicular transport of proteins from the ER.

\section{Osh proteins function as negative regulators of vesicle budding from the ER}

Although Osh proteins do not have an essential function in protein trafficking, they might act as negative regulators of protein transport. Indeed, deletion of $\mathrm{OSH} 4$ has been reported to suppress the Golgi secretory defect in sec14 mutant cells (Fang et al., 1996). To investigate the possibility that Osh proteins may regulate other secretory processes, we created strains carrying multiple deletions of $\mathrm{OSH}$ genes in different sec mutants and examined the growth phenotypes at various temperatures. Consistent with previous studies (Fang et al., 1996), deletions of $\mathrm{OSH} 4$ were able to suppress the lethality associated with a mutation in the SEC14 gene (Fig. 3A). Interestingly, a triple deletion of $\mathrm{OSH} 2, \mathrm{OSH} 3$ and $\mathrm{OSH} 4$ and, less efficiently, a double deletion of the $\mathrm{OSH} 2$ and $\mathrm{OSH} 4$ rescued the severe growth defect of sec 12-4 mutant cells at semi-permissive temperatures $\left(33^{\circ} \mathrm{C}\right.$ and $35^{\circ} \mathrm{C}$ ). A partial rescue was also seen in sec $23-1$ or sec $16-2$ mutant cells lacking three $\mathrm{OSH}$ genes (Fig. 3B); however, rescue was not observed in other sec mutant (sec31-1, sed5-1, sec18-20, ret $1-1$ and sec35-1) strains. Because Sec12p regulates the initiation of COPII vesicle budding from the ER and Sec23p is a component of the COPII vesicle coat (Miller and Barlowe, 2010; Sato and Nakano, 2007), our data strongly suggest that Osh proteins function as negative regulators of vesicle budding from the ER.

To assess more directly the negative role of Osh proteins in COPII vesicle budding, we first analyzed the maturation of cargo proteins, Gas1p and CPY. We found that the delayed kinetics of maturation of Gas1p and CPY in sec12-4 mutant cells were partially suppressed by the triple deletion of $\mathrm{OSH} 2, \mathrm{OSH} 3$ and $\mathrm{OSH} 4$ at a semi-permissive temperature $\left(33^{\circ} \mathrm{C}\right.$; Fig. $\left.4 \mathrm{~A}\right)$, whereas no suppression was observed when pulse-chase experiments were carried out at higher temperature $\left(35^{\circ} \mathrm{C}\right.$; data not shown). Next, we examined the localization of Rerlp, which is a retrieval receptor for ER membrane proteins and is recycled between the ER and Golgi but is localized in the Golgi at steady-state (Sato et al., 2001). When the ER to Golgi anterograde transport is blocked by the sec12-4 mutation, GFP-Rerlp accumulates in the ER (Sato et al., 2001). If anterograde transport by COPII vesicles is negatively regulated by Osh proteins, the osh $2 \Delta \operatorname{osh} 3 \Delta \operatorname{osh} 4 \Delta$ sec12-4 quadruple mutant should not accumulate GFP-Rer1p in the ER, but should instead accumulate it in the Golgi. Indeed, upon a shift to semi-permissive temperatures $\left(35^{\circ} \mathrm{C}\right)$, the sec $12-4$ mutant caused an increase in the number of cells with GFP-Rerlp localized to the ER only (nuclear ring staining) but not to the Golgi (punctate staining). The osh2 $\Delta$ osh34 osh $4 \Delta$ sec12-4 mutant showed no significant impact on the localization of GFPRerlp, in comparison with those of the strains at permissive temperature $\left(25^{\circ} \mathrm{C}\right.$; Fig. $\left.4 \mathrm{~B}, \mathrm{C}\right)$. These results suggest that the anterograde transport defects in the sec12-4 mutant are at least partially rescued by the absence of Osh2p, Osh3p and Osh4p.

Because Sec12p seems to be implicated in the biogenesis of ER exit sites (ERES), where budding of the COPII vesicles occurs (Castillon et al., 2009; Shindiapina and Barlowe, 2010), we next investigated whether deletion of $\mathrm{OSH}$ genes affects the 
A

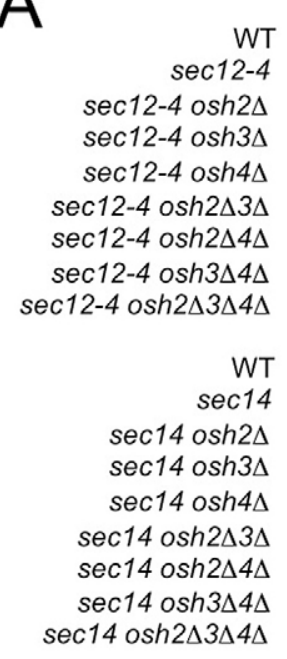

B

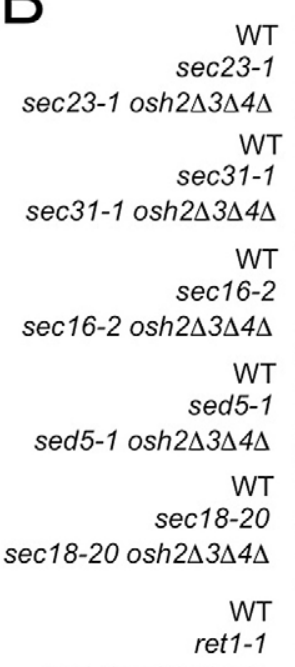

ret1-1 osh $2 \Delta 3 \Delta 4 \Delta$

W
ret1-1
$3 \Delta 4 \Delta$

WT

$\sec 35-1$

$\sec 35-1 \operatorname{osh} 2 \Delta 3 \Delta 4 \Delta$ $25^{\circ} \mathrm{C}$

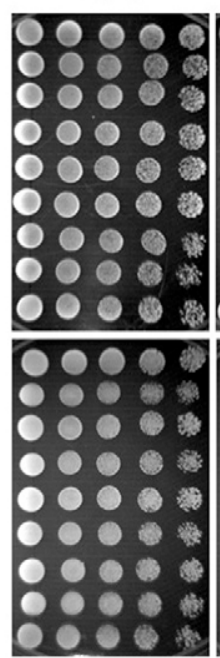

$30^{\circ} \mathrm{C}$
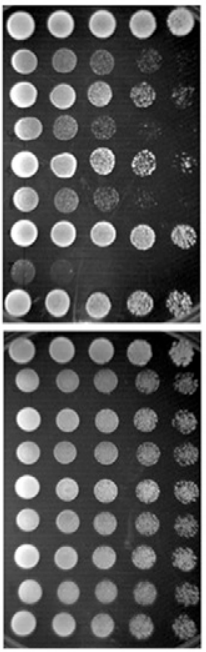

$30^{\circ} \mathrm{C}$

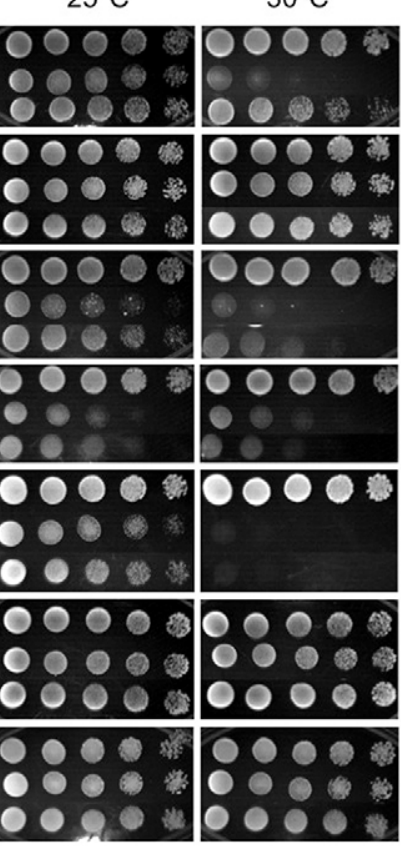

$33^{\circ} \mathrm{C}$

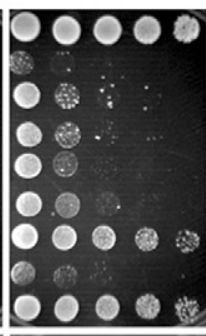

$35^{\circ} \mathrm{C}$
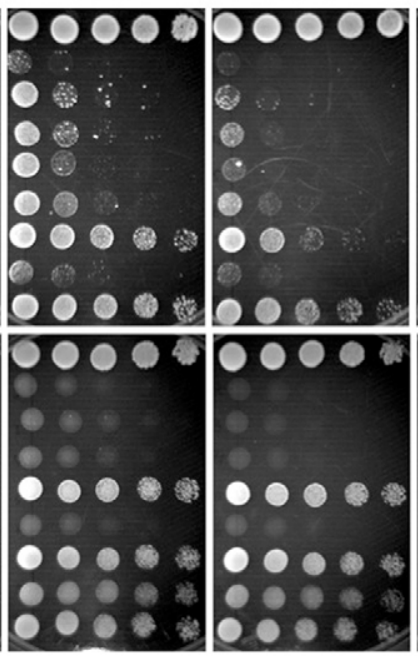

$37^{\circ} \mathrm{C}$
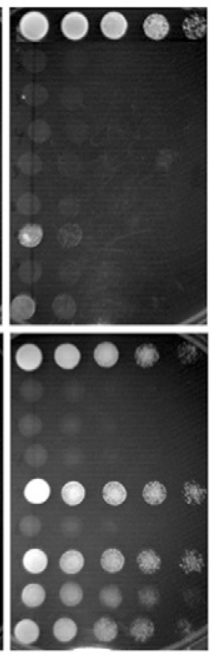

Fig. 3. Triple deletion of $\mathrm{OSH} 2$, $\mathrm{OSH} 3$ and $\mathrm{OSH} 4$ suppresses the temperature-sensitive growth defects of sec12-4 and sec23-1 mutants. (A,B) Fivefold serial dilutions of cells were spotted onto SD plates and the plates were then incubated at the indicated temperatures for 3 days.

localization of the ERES marker Sec13p in sec12-4 mutant cells. The number of Sec13p-mCherry puncta per sec12-4 mutant cell was significantly $(P<0.01)$ reduced (Fig. 4D,E), whereas the number of cells with large dots of Sec13p-mCherry was strongly increased (Fig. 4D,F) even at a semi-permissive temperature $\left(30^{\circ} \mathrm{C}\right)$. These relatively mild phenotypes observed at $30^{\circ} \mathrm{C}$ were also rescued by the deletion of $\mathrm{OSH} 2, \mathrm{OSH}_{3}$ and $\mathrm{OSH} 4$ (Fig. 4DF), suggesting that Osh proteins negatively regulate the organization of ERES and thus the vesicle budding from the ER.

\section{DISCUSSION}

Our results support the view that whereas Osh proteins play a role in the positive regulation of vesicular transport of ceramide from the ER to the Golgi, they negatively regulate COPII-mediated ER export of cargoes. How Osh proteins regulate these processes is unknown. However, our observations showed that loss of either $\operatorname{Scs} 2 \mathrm{p}$ alone or both $\mathrm{Scs} 2 \mathrm{p}$ and $\mathrm{Scs} 22 \mathrm{p}$, which localize to ER and bind to FFAT (two phenylalanines in an acidic tract) motifs of Osh proteins to target them to the ER (Loewen et al., 2003), did not affect the synthesis of IPC-C (supplementary material Fig. S4A,B), whereas the loss did result in partial suppression of the temperature-sensitive growth phenotype of sec12-4 mutant (supplementary material Fig. S4C). We therefore propose the following model for the two roles of Osh proteins (Fig. 5A). When bound to $S \operatorname{cs} 2 p$ and/or Scs $22 p$ and/or the ER, Osh proteins negatively regulate cargo sorting or vesicle scission at ER exit sites. When Osh proteins dissociate from the ER, they positively regulate or facilitate the targeting of ceramide-enriched vesicles to the Golgi, where they are converted into IPC.

In budding yeast, ceramides and GPI-APs mutually regulate their vesicular transport from the ER to the Golgi (Horvath et al., 1994; Kajiwara et al., 2008; Watanabe et al., 2002), leading to the proposal that ceramides and GPI-APs are co-transported in the same vesicles budding from the ER (Kajiwara et al., 2008). 

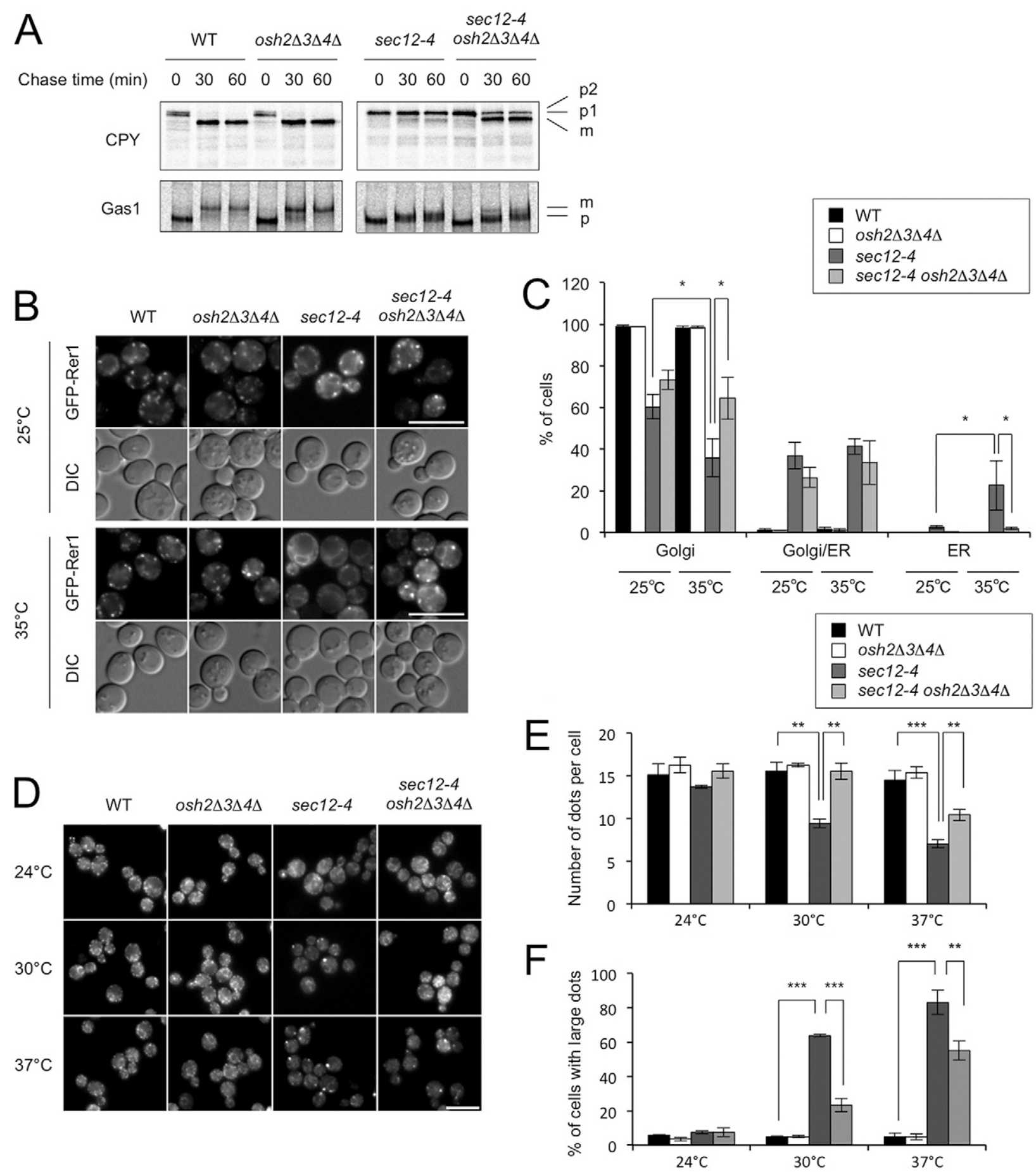

Fig. 4. Triple deletion of $\mathrm{OSH} 2, \mathrm{OSH} 3$ and $\mathrm{OSH} 4$ significantly rescues the transport defects in the sec12-4 mutant. (A) Cells were labeled with $\left[{ }^{35} \mathrm{~S}\right]$ methionine for 5 minutes and chased at $33^{\circ} \mathrm{C}$ for the indicated times. Proteins were immunoprecipitated, resolved by SDS-PAGE, and analyzed with a phosphoimager. (B,C) Cells expressing GFP-Rer1p were treated with cycloheximide $(0.2 \mathrm{mg} / \mathrm{ml})$ at $25^{\circ} \mathrm{C}$ for 20 minutes and then incubated at $25^{\circ} \mathrm{C}$ or $35^{\circ} \mathrm{C}$. After 60 minutes, localization of GFP-Rer1p was observed by fluorescence microscopy and the images were acquired within 10 minutes (B). The subcellular localization of GFP-Rer1p was quantified by counting the number of cells that displayed nuclear ring staining (ER), punctate staining (Golgi) or both (C). More than 200 cells were evaluated for each sample per experiment, and data are means \pm s.d. $(n=3)$ of three independent experiments. ${ }^{*} P<0.05$. (D-F) Cells expressing Sec13pmCherry were incubated at the indicated temperatures for 60 minutes and observed by fluorescence microscopy (D). Fluorescence micrographs acquired within 10 minutes were used to quantify the number of fluorescent dots per cell $(E)$ and the number of cells with large dots $(F)$. At least 30 cells were evaluated for each sample per experiment, and data are means \pm s.d. $(n=3)$ of three independent experiments. ${ }^{* \star} P<0.01 ;{ }^{* \star *} P<0.001$. Scale bars: $10 \mu \mathrm{m}$.

Because vesicular transport of ceramide from the ER is mediated by COPII-coated vesicles (Funato and Riezman, 2001), if ceramides are not directly recognized by COPII coat proteins, other cargo-cargo receptor complexes that are recognized by COPII coat proteins must be incorporated at the ERES where ceramide-rich vesicles are formed. The p24 proteins have been proposed to act as cargo receptors for GPI-APs to be sorted into COPII vesicles (Castillon et al., 2011; Gillon et al., 

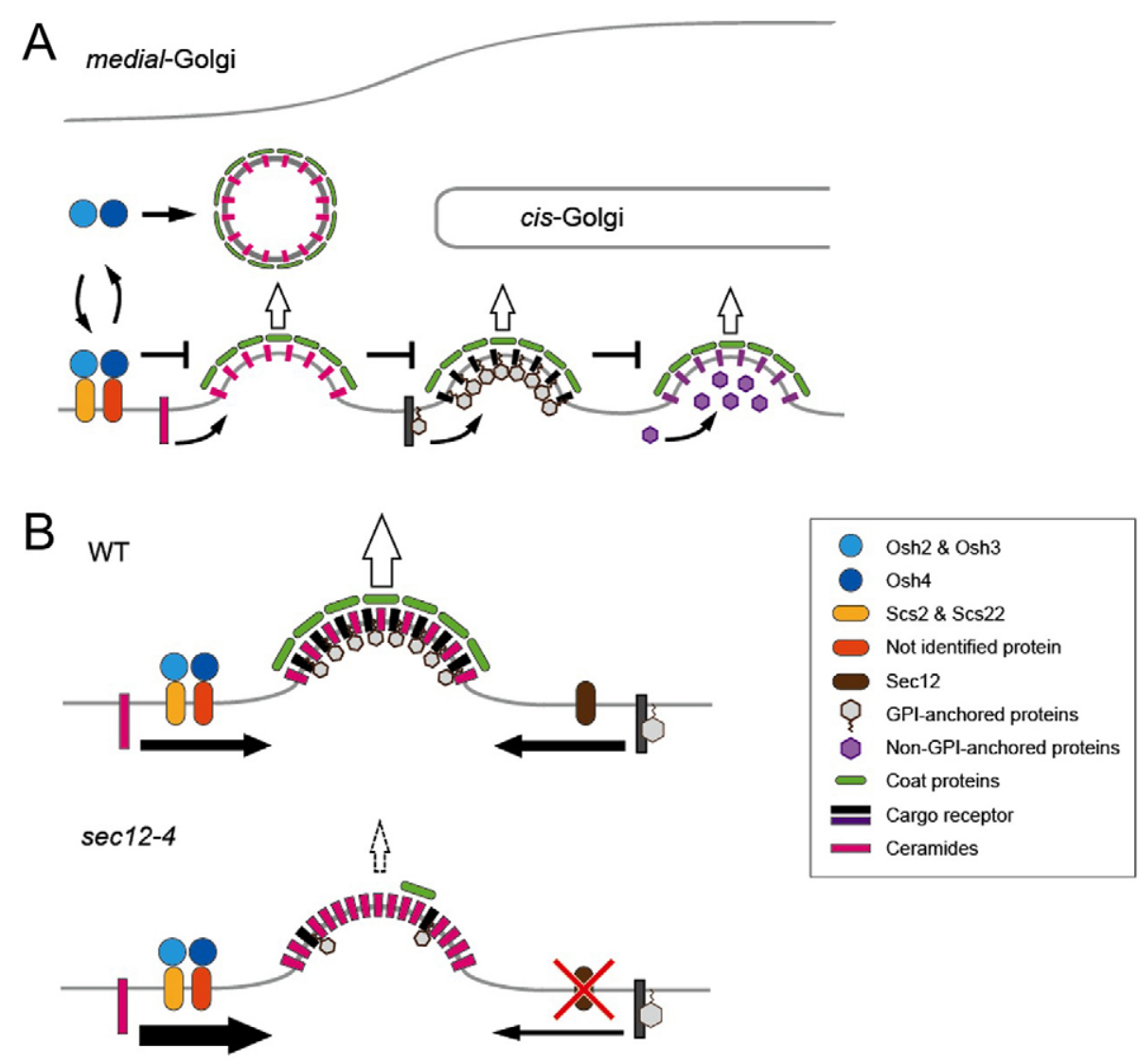

Fig. 5. Models for the function of Osh proteins in vesicular traffic from the ER. See Discussion for further explanation.

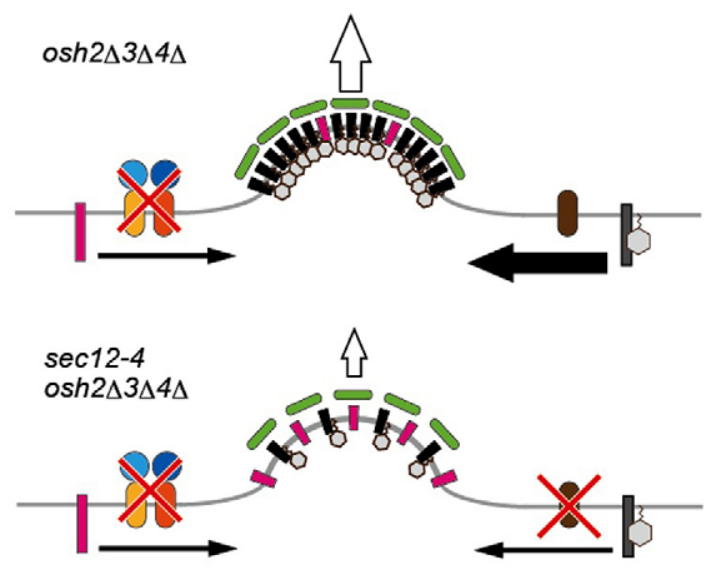

2012). In the case in which ceramides and GPI-APs are incorporated into the same vesicles, it is tempting to consider the possibility that Osh proteins may promote concentration of ceramide at ERES (Fig. 5B). This could lead to an excess concentration of ceramides at Sec12p-deficient ERES that interferes with the incorporation of GPI-APs and the cargoprotein-mediated COPII assembly. In cells lacking Osh proteins, a sufficient COPII assembly to form vesicles containing cargo proteins, even without Sec12p, may be achieved by blocking concentration of ceramides at ERES. According to this partitioning model, double deletions of $\mathrm{OSH} 2$ and $\mathrm{OSH} 4$ or SCS 2 and SCS22 would cause decreased IPC synthesis because they have sufficient ability to rescue the temperature-sensitive growth defect of sec12-4 mutant (Fig. 3A; supplementary material Fig. S4C). However, the osh $2 \Delta \operatorname{osh} 4 \Delta$ and $\operatorname{scs} 2 \Delta$ scs 224 mutants did not show any decrease in IPC synthesis (supplementary material Fig. S1A; Fig. S4A,B). Additionally, the partitioning model cannot explain adequately why deletion of $\mathrm{OSH} 2, \mathrm{OSH} 3$ and $\mathrm{OSH} 4$ rescues not only the GPI-AP transport defect, but also the non-GPI-AP transport defects of sec12-4 mutant because the transport of ceramides and GPI-APs do not seem to be coupled to that of non-GPI-APs (Kajiwara et al., 2008).

Although we cannot entirely rule out the possibility that Osh proteins facilitate the packaging of ceramide into GPI-APsenriched COPII vesicles, and other models are also conceivable, a specific requirement for Osh proteins in ceramide trafficking suggests that ceramide-enriched vesicles could be distinct from those containing GPI-APs (Fig. 5A). This raises the following questions: what is the role of the specific vesicle that contains 
ceramides and the role of Osh proteins in ceramide delivery to the Golgi compartment? In this connection, it is interesting to note that the IPC synthase catalytic subunit, Aurlp, is localized mainly to the medial-Golgi but not the cis-Golgi (Levine et al., 2000). Because the domains of Osh proteins such as pleckstrin homology domain, FFAT and ORP-related domain motifs have the ability to form the contact sites between the ER and the Golgi (Beh et al., 2012; Schulz et al., 2009; Vihervaara et al., 2011), Osh proteins may serve as tethering factors to target ER-derived ceramide vesicles directly to the medial-Golgi or as bridging proteins to facilitate ceramide delivery by forming unique regions of the ER membrane closely apposed to the medial-Golgi, reducing the distance between the organelles. Otherwise Osh proteins could generate a membrane environment leading to specific targeting of ceramide-enriched vesicles because of the ability of some Osh proteins to exchange lipids between lipid bilayers (Beh et al., 2012; Schulz et al., 2009; Vihervaara et al., 2011). Although this unconventional process and its mechanistic basis remain to be elucidated, it may play a crucial role in maintaining the metabolism and distribution of sphingolipids, which can be involved in diverse cellular functions such as signal transduction, membrane trafficking and other lipid metabolism.

The reason for specific lipid requirements in efficient transport of GPI-APs also remains unknown, but it is conceivable that a specific lipid environment on Golgi membranes may be required for docking and fusion of GPI-AP-enriched vesicles, or that the role of ceramides in GPI-AP transport is solely in anchor remodeling (Horvath et al., 1994; Watanabe et al., 2002).

Our findings that deletion of the $\mathrm{OSH} 2, \mathrm{OSH} 3$ and $\mathrm{OSH} 4$ genes rescues the phenotypes of sec12-4 mutant cells are surprising. Given that Osh2p and Osh3p but not Osh4p have a FFAT motif (Loewen et al., 2003), that the suppressive effect of scs 2 mutation on growth of sec12-4 is enhanced by OSH4 deletion (supplementary material Fig. S4C), which is specific to sec12-4 (supplementary material Fig. S4D) and dependent on the association with the FFAT motif (supplementary material Fig. S4E), it is probable that the bypass of SEC12 function by inactivation of three $\mathrm{OSH}$ genes involves both $\mathrm{Scs} 2 \mathrm{p}-\mathrm{Scs} 22 \mathrm{p}$ FFAT-dependent and -independent modes (Fig. 5A). According to this and the model for two roles of Osh proteins in vesicle budding and targeting, the $\operatorname{scs} 2 \Delta \operatorname{scs} 22 \Delta$ mutant would have enhanced ceramide transport. However, this does not seem to be the case (supplementary material Fig. S4A,B). Osh4p does not have FFAT motifs and it may be associated with an unidentified ER-resident protein. Thus, cytosolic Osh4p might be necessary to stimulate the targeting of ceramide-enriched vesicles to the Golgi compartment.

Mechanistic details of the bypass of SEC12 function are unknown; however, there are several possibilities that can be considered. One possibility is that the bypass of SEC12 function could result from reduced levels of GPI-APs as the membranedeforming ability of the COPII coats might depend on the local concentration of luminal-asymmetric membrane cargoes (Čopič et al., 2012). But, this seems unlikely because triple null mutations (osh2A osh3A osh4A) did not impact Gas1p maturation and because the temperature-sensitive phenotype of the sec12-4 mutant was not bypassed by loss of ERII, which functions in GPI synthesis (data not shown). Another possibility, that activation of unfolded protein response, which reduces the protein load in the ER lumen (Chang et al., 2004), contributes to the bypass of SEC12 function is also unlikely. Indeed, we found that unfolded protein response-dependent expression of a yeast chaperone, Kar2p was not affected in osh 24 osh 34 osh $4 \Delta$ cells (data not shown).

COPII-mediated ER export, from coat recruitment to vesicle scission, depends on the membrane composition, not only of proteins but also lipids (Blumental-Perry et al., 2006; Farhan and Rabouille, 2011; Miller and Barlowe, 2010; Shindiapina and Barlowe, 2010). Yeast cells lacking Osh proteins or both Scs2p and $\operatorname{Scs} 22 \mathrm{p}$ accumulate PtdIns4P (Stefan et al., 2011). It has been reported that in mammalian cells, PtdIns $4 P$ at ERES is required to regulate membrane deformation at ERES and promote COPIImediated ER export (Blumental-Perry et al., 2006). However, the analysis of mutants revealed that alterations in PtdIns $4 P$ metabolism did not impact ER export in in vitro budding yeast (Audhya et al., 2000; Lorente-Rodríguez and Barlowe, 2011). Consistent with this, the results from a previous study (Cleves et al., 1989) and this study (supplementary material Fig. S3C) showed that the temperature-sensitive growth defect of the sec12-4 mutant was not suppressed by SAC1 mutations, suggesting that the ability of osh $\Delta$ to suppress sec12-4 mutant phenotypes does not depend on Sac1p.

In addition, it is unlikely that the suppression of the protein transport defect of the sec12-4 by deletions of $\mathrm{OSH}$ genes is due to an altered sphingolipid metabolism. Two observations support this. The osh $2 \Delta$ osh $4 \Delta$ double and osh $2 \Delta \operatorname{osh} 3 \Delta$ osh $4 \Delta$ triple deletions that can suppress the growth defect of sec12-4 had different effects on sensitivity to $\mathrm{AbA}$ and on IPC synthesis, suggesting that the ability to suppress does not correlate with the changes in sphingolipid levels. We also found that the growth phenotype of the sec12-4 mutant was not rescued by AbA ( $0.05 \mu \mathrm{g} / \mathrm{ml}$; data not shown) or Myr $(0.1 \mu \mathrm{g} / \mathrm{ml}$; data not shown $)$ or treatment by supplementation with ergosterol $(100 \mu \mathrm{g} / \mathrm{ml}$; data not shown). These results imply that neither altered sphingolipids nor sterols contribute to the bypass of SEC12 function. Furthermore, our findings that $\operatorname{scs} 2 \Delta$ but not $\operatorname{osh} 2 \Delta \operatorname{osh} 3 \Delta$ osh $4 \Delta$ mutant is an inositol auxotroph (supplementary material Fig. S4F) indicate that the ability of $O S H$ deletion to rescue sec 12-4 phenotypes might be not due to an impaired regulation of phospholipid synthesis.

Although the current study does not completely elucidate how Osh proteins regulate COPII-mediated ER export, they might act as a sensor to control the GTP-GDP cycle of the small GTPase Sarlp, which is regulated by the guanine nucleotide exchange factor, Sec12p and the GTPase-activating protein, Sec23p. This idea is consistent with our results showing that loss of Osh proteins can rescue both temperature-sensitive phenotypes of sec12-4 and sec23-1 mutants. In addition to functioning in the assembly and disassembly of the COPII coat, the GTP-GDP cycle of Sarlp seems to be involved in vesicle scission (Sato and Nakano, 2007). The continual GTP-GDP cycles would lead to an efficient but also incorrect vesicle scission. Thus, Osh proteins possibly retard the abnormal cycles to prevent disordered vesicle scission that forms vesicles carrying mis-sorted cargoes. The potential involvement of the GTP-GDP cycle in cargo selection and concentration has been proposed (Miller and Barlowe, 2010; Sato and Nakano, 2007), so it might also be that Osh proteins function to avoid the excess or improper loading of cargoes such as mis-sorted, misfolded and ER-resident proteins into the vesicles, perhaps coordinated with Sec16p, which regulates the GTP-GDP cycle of Sar1p and organization of ERES (Kung et al., 2012; Miller and Barlowe, 2010). However, our experiments indicated that triple deletion of $\mathrm{OSH} 2, \mathrm{OSH} 3$ and $\mathrm{OSH} 4$ was not able to rescue cytoplasmic mislocalization of Sarlp-mCherry in 
sec12-4 mutants (data not shown). This suggests that Osh proteins are not involved in regulating the GTP-GDP cycle of Sarlp because Sar1p localization is dependent on the nucleotidebinding state (Yorimitsu and Sato, 2012). Thus, it is possible that Osh proteins play a role in coat stabilization after GTP hydrolysis.

Finally, we favor the model with two distinct functional roles of Osh proteins in vesicular transport of ceramide to the Golgi and COPII-mediated ER export. As ceramides might be sorted into COPII vesicles different from those for proteins, and targeted to the Golgi compartments by a distinct molecular mechanism that involves Osh proteins, further study of the mechanism will provide new insights not only into the sorting process at ERES but also into the establishment and maintenance of intracellular sphingolipid levels.

\section{MATERIALS AND METHODS \\ Strains and plasmids}

All yeast strains used in this study are listed in supplementary material Table S1. Yeast strains were generated either by PCR-based one-step gene replacement (Baudin et al., 1993) or by crossing, sporulation and subsequent dissection of the spores. Deletion strains were confirmed by PCR. C-terminal triple hemagglutinin (HA)-tagging of Osh4p (Osh4p$3 \times \mathrm{HA})$ was carried out by a PCR-based method to generate the FKY146 strain from FKY136 as described previously (Levine et al., 2000). DNA cloning techniques, Escherichia coli transformation and site-directed mutagenesis were performed as described previously (Kagiwada and Hashimoto, 2007; Sambrook et al., 1989) and by standard procedures. The plasmid for expression of Osh $4 \mathrm{p}-3 \times \mathrm{HA}$ was constructed as follows. The BamHI-SalI fragment containing the open reading frame of $O S H 4-3 \times \mathrm{HA}$ was amplified by PCR using the FKY146 strain as template and cloned into pRS415ADH (CEN, LEU2), which contains the $A D H 1$ promoter and the $C Y C 1$ terminator, to obtain pRS415ADH-OSH4-3 $\times$ HA (FKP161). To generate plasmid pcDNAOSH4-3 $\times$ HA (FKP117), the BamHI-SalI fragment containing OSH4-3 $\times$ HA from pRS415ADH-OSH4-3 $\times$ HA was inserted into the Bam HI-XhoI sites of pcDNA3.1(+)Neo. The plasmid to express HAtagged human CERT (HA-hCERT) in yeast was made from pcDNA-HAhCERT (Kudo et al., 2008). The EcoRI-XhoI fragment containing HA-hCERT was inserted into pRS414GPD (which contains the TDH3 promoter) and subcloned into $S m a \mathrm{I}-\mathrm{XhoI}$ sites of pRS415ADH to obtain pRS415ADH-HA-hCERT (FKP171). The XbaI-XhoI fragments containing OSH4-3×HA from FKP161 and HA-hCERT from FKP171 were also subcloned into $X b a \mathrm{I}-X h o \mathrm{I}$ sites of pRS416GPD to obtain pRS416GPD-OSH4-3 $\times$ HA (FKP353) and pRS416GPD-HA-hCERT (FKP353), respectively. A LEU2 plasmid expressing Sec13p-mCherry (YCplac111-Sec13-mCh) was constructed as described previously (Castillon et al., 2011), by PCR using the primers 5'-gggatatcaggaggcttccgagattttgg-3' and $5^{\prime}$-gggatatcctcgcaggtctgcagcgaggcgcc-3' followed by enzymatic digestion with $E c o R V$ and ligation. To generate LEU2 plasmids expressing wild-type Scs2 (pRS415-SCS2), mutant scs2 K[80/84/119/122]A (pRS415-scs2-K[80/84/119/122]A) and L84D/L86A (pRS415-scs2-L84D/L86A) under its own promoter, the fragments containing wild-type SCS2 from YCplac33-SCS2 (pKY151) (Kagiwada et al., 1998) and mutant scs2 from YCplac33-mutant scs2 vectors, respectively, which were constructed as described previously (Kagiwada and Hashimoto, 2007), were subcloned into HindIII-BamHI sites of pRS415. A LEU2 plasmid expressing GFP-tagged Rer1p (pRS415-GFPRER1) was constructed from pSKY5-RER1 (Sato et al., 2001), which was kindly provided by A. Nakano (RIKEN, Japan). The BamHI-SalI fragment containing the $T D H 3$ promoter, the open reading frame of GFPRERI and the CMK1 terminator were subcloned into BamHI-SalI sites of pRS415. The pRS425-SAC1 plasmid was constructed by subcloning $S A C l$ as a $2.09-\mathrm{kb} K p n \mathrm{I}-\mathrm{SacI}$ genomic DNA fragment that includes the $S A C 1$ promoter and $S A C 1$ gene from YEplac195-SACl (Kagiwada et al., 1996) into pRS425.

\section{Drug and temperature sensitivity assays}

Drug and temperature sensitivity assays were performed as described previously (Kajiwara et al., 2012). Briefly, cells were grown to log phase at $25^{\circ} \mathrm{C}$ in synthetic dextrose (SD) minimal medium (Funato et al., 2003). The next day cells at an initial concentration of $\mathrm{OD}_{600 \mathrm{~nm}}=1$ were serially diluted 1:5 in sterile water. Eight microliters of each 5-fold serial dilution was spotted onto SD plates containing the indicated concentrations of Myr (BIOMOL Research Laboratories Inc., PA, USA) or AbA (Takara Bio Inc., Shiga, Japan) and incubated at $25^{\circ} \mathrm{C}$ for 3 days. Temperature sensitivity was carried out by spotting diluted yeast cultures on SD plates supplemented with the appropriate nutrients to select for plasmids and by incubating at the indicated temperatures.

\section{Lipid labeling}

Labeling of yeast sphingolipids with $\left[{ }^{3} \mathrm{H}\right]$ myo-inositol (PerkinElmer Life Sciences) or $\left[{ }^{3} \mathrm{H}\right] \mathrm{DHS}$ (American Radiolabeled Chemical Inc.) was performed as described previously (Kajiwara et al., 2008). Cells grown overnight in SD medium at $25^{\circ} \mathrm{C}$ were resuspended in SD without inositol for labeling with $\left[{ }^{3} \mathrm{H}\right] m y o$-inositol, or SD medium for labeling with $\left[{ }^{3} \mathrm{H}\right] \mathrm{DHS}$, preincubated at the indicated temperatures for 20 minutes, and then labeled with $10 \mu \mathrm{Ci}\left[{ }^{3} \mathrm{H}\right]$ myo-inositol or $4 \mu \mathrm{Ci}\left[{ }^{3} \mathrm{H}\right] \mathrm{DHS}$ at the same temperature used for preincubation, for the indicated times. If present, $50 \mu \mathrm{M} \mathrm{C}_{2}$-ceramide (Avanti Polar Lipids, Inc. AL, USA) was added before the labeling. For the energy deprivation experiments (Funato and Riezman, 2001), cells were preincubated on ice for 30 minutes and were pulse labeled with $20 \mu \mathrm{Ci}$ of $\left[{ }^{3} \mathrm{H}\right] m y o$-inositol on ice for 60 minutes. After addition of $100 \mu \mathrm{g} / \mathrm{ml}$ cold myo-inositol, $10 \mathrm{mM} \mathrm{NaF}$ and $10 \mathrm{mM}$ $\mathrm{NaN}_{3}$ were added to the cells followed by incubation on ice for 10 minutes, the energy-deprived cells were chased by adding ten times the volume of SD medium containing myo-inositol $(10 \mu \mathrm{g} / \mathrm{ml})$, at $25^{\circ} \mathrm{C}$ for 60 minutes. The reaction was stopped by placing the mixture on ice. The cells were washed with cold water and subjected to lipid extraction, and if necessary, to mild alkaline hydrolysis to deacylate glycophospholipids as described previously (Kajiwara et al., 2008). The pooled organic phases were desalted by partitioning with $n$-butanol, and lipids were dried under nitrogen. Radiolabeled lipids were analyzed by thin layer chromatography (TLC) using solvent system I, chloroform:methanol: $0.25 \% \mathrm{KCl}(55: 45: 10, \mathrm{v} / \mathrm{v})$ for lipids labeled with $\left[{ }^{3} \mathrm{H}\right]$ myo-inositol, solvent system II: chloroform:methanol:4.2 $\mathrm{N}$-ammonium hydroxide $(9: 7: 2, \mathrm{v} / \mathrm{v})$ for lipids labeled with $\left[{ }^{3} \mathrm{H}\right] \mathrm{DHS}$, and were quantified using the FLA-7000 system (Fujifilm). For ceramide analysis, the lipids labeled with $\left[{ }^{3} \mathrm{H}\right] \mathrm{DHS}$ were separated on TLC plates using solvent system II. Subsequently, fractions containing ceramides, which are $\sim 2-3 \mathrm{~cm}$ from the top of the TLC plates (Funato and Riezman, 2001; Kajiwara et al., 2012), were collected by scraping and eluting with chloroform:methanol $(1: 1, \mathrm{v} / \mathrm{v})$, and were analyzed by TLC using solvent system III: chloroform:methanol:acetc acid (190:9:1, v/v), as described previously (Cremesti and Fischl, 2000; Haak et al., 1997).

Metabolic labeling of $\mathrm{CHO}$ cells with $\left[{ }^{14} \mathrm{C}\right]$ serine was performed as described previously (Kawano et al., 2006). Briefly, cell lines CHO-K1 and LY-A, a CHO-KI-derived mutant cell line defective in ER-to-Golgi transport of ceramide (Hanada et al., 2003), which was transiently transfected with plasmids using Lipofectamine Plus ${ }^{(i)}$ reagent (Invitrogen) were grown and incubated in Nutridoma medium containing $\left[{ }^{14} \mathrm{C}\right]$ serine at $37^{\circ} \mathrm{C}$ for 2 hours. After two washes with cold phosphate-buffered saline, labeled lipids were extracted from the cells and were analyzed by TLC using solvent system IV: methyl acetate: $n$ propanol:chloroform:methanol:0.25\% $\mathrm{KCl}$ (25:25:25:10:9, v/v).

\section{Protein transport assays}

Pulse-chase analysis of Gas1p and CPY was carried out exactly as described (Horvath et al., 1994; Watanabe et al., 2002). The pRS415GFP-RERI plasmid carrying GFP-tagged Rer1p was used for a trafficking assay of a cargo receptor. Yeast strains transformed with pRS415-GFP-RER1 were treated with cycloheximide $(0.2 \mathrm{mg} / \mathrm{ml})$ at $25^{\circ} \mathrm{C}$ for 20 minutes prior to incubation at the indicated temperatures for 60 minutes, and observed by fluorescence microscopy (Olympus BX51, Tokyo, Japan). In order to observe ER exit sites where newly synthesized 
proteins are packaged into budding COPII vesicles, cells were transformed with YCplac111-Sec13-mCh and were observed using a AXIOZ1 microscope as described previously (Castillon et al., 2011).

\section{Immunoblotting}

Protein extraction from yeast, SDS-PAGE and western blotting were performed as described previously (Schönbächler et al., 1995). For protein extraction from $\mathrm{CHO}$ cells, the cells cultured in dishes, were washed with phosphate-buffered saline and harvested in buffer A (40 mM Tris- $\mathrm{HCl}, \mathrm{pH} 7.4,180 \mathrm{mM} \mathrm{NaCl}$ and $1 \mathrm{mM}$ EDTA) by scraping (Tomishige et al., 2009). After precipitation by centrifugation, cells were suspended in buffer B [10 mM HEPES-NaOH, pH 7.4, $250 \mathrm{mM}$ sucrose, $1 \mathrm{mM}$ EDTA and protease inhibitor cocktail (Complete; Roche Diagnostics)], and lysed by sonication. The cell lysate was subjected to SDS-PAGE and western blotting. Blots were probed with a rat monoclonal antibody against HA (3F10) and a peroxidase-conjugated affinity-purified anti-rat $\operatorname{IgG}$ antibody.

\section{Acknowledgements}

We thank Christopher Beh and Randy Schekman for providing strains, Aki Nakano for the pSKY5-RER1 plasmid and Ken Sato for the SAR1-mCherry plasmids. We also thank Lawrence M. Liao for proofreading the manuscript.

\section{Competing interests}

The authors declare no competing interests.

\section{Author contributions}

K.K., A.I., A.A.-R., G.A.C. and K.H. performed cellular and biochemical experiments; S.K. constructed the plasmids; H.R., M.M. and K.F. provided technical advice, designed the experiments and wrote the paper with comments from co-authors.

\section{Funding}

This work was supported by the ONO Medical Research Foundation [to K.F.]; Research Fellowships from the Japan Society for the Promotion of Science for Young Scientists [grant number $08 J 04662$ to K.K.]; the Spanish Ministry of Science and Innovation [grant number BFU2011-24513 to M.M.]; and the Junta de Andalucía [grant number P09-CVI-4503 to M.M.].

\section{Supplementary material}

Supplementary material available online at

http://jcs.biologists.org/lookup/suppl/doi:10.1242/jcs.132001/-/DC1

\section{References}

Audhya, A., Foti, M. and Emr, S. D. (2000). Distinct roles for the yeast phosphatidylinositol 4-kinases, Stt4p and Pik1p, in secretion, cell growth, and organelle membrane dynamics. Mol. Biol. Cell 11, 2673-2689.

Baudin, A., Ozier-Kalogeropoulos, O., Denouel, A., Lacroute, F. and Cullin, C. (1993). A simple and efficient method for direct gene deletion in Saccharomyces cerevisiae. Nucleic Acids Res. 21, 3329-3330.

Beh, C. T. and Rine, J. (2004). A role for yeast oxysterol-binding protein homologs in endocytosis and in the maintenance of intracellular sterol-lipid distribution. J. Cell Sci. 117, 2983-2996.

Beh, C. T., Cool, L., Phillips. J. and Rine, J. (2001). Overlapping functions of the yeast oxysterol-binding protein homologues. Genetics 157, 1117-1140.

Beh, C. T., McMaster, C. R., Kozminski, K. G. and Menon, A. K. (2012). A detour for yeast oxysterol binding proteins. J. Biol. Chem. 287, 11481-11488.

Blumental-Perry, A., Haney, C. J., Weixel, K. M., Watkins, S. C., Weisz, O. A. and Aridor, M. (2006). Phosphatidylinositol 4-phosphate formation at ER exit sites regulates ER export. Dev. Cell 11, 671-682.

Brice, S. E., Alford, C. W. and Cowart, L. A. (2009). Modulation of sphingolipid metabolism by the phosphatidylinositol-4-phosphate phosphatase Sac1p through regulation of phosphatidylinositol in Saccharomyces cerevisiae. J. Biol. Chem. 284, 7588-7596.

Castillon, G. A., Watanabe, R., Taylor, M., Schwabe, T. M. and Riezman, H. (2009). Concentration of GPI-anchored proteins upon ER exit in yeast. Traffic $10,186-200$.

Castillon, G. A., Aguilera-Romero, A., Manzano-Lopez, J., Epstein, S., Kajiwara, K., Funato, K., Watanabe, R., Riezman, H. and Muñiz, M. (2011). The yeast p24 complex regulates $\mathrm{GPl}$-anchored protein transport and quality control by monitoring anchor remodeling. Mol. Biol. Cell 22, 2924-2936.

Chang, H. J., Jesch, S. A., Gaspar, M. L. and Henry, S. A. (2004). Role of the unfolded protein response pathway in secretory stress and regulation of INO1 expression in Saccharomyces cerevisiae. Genetics 168, 1899-1913.

Cleves, A. E., Novick, P. J. and Bankaitis, V. A. (1989). Mutations in the SAC1 gene suppress defects in yeast Golgi and yeast actin function. J. Cell Biol. 109, 2939-2950.
Čopič, A., Latham, C. F., Horlbeck, M. A., D'Arcangelo, J. G. and Miller, E. A. (2012). ER cargo properties specify a requirement for COPII coat rigidity mediated by Sec13p. Science 335, 1359-1362.

Cremesti, A. E. and Fischl, A. S. (2000). Current methods for the identification and quantitation of ceramides: an overview. Lipids 35, 937-945.

de Saint-Jean, M., Delfosse, V., Douguet, D., Chicanne, G., Payrastre, B., Bourguet, W., Antonny, B. and Drin, G. (2011). Osh4p exchanges sterols for phosphatidylinositol 4-phosphate between lipid bilayers. J. Cell Biol. 195, 965-978.

Epstein, S., Castillon, G. A., Qin, Y. and Riezman, H. (2012). An essential function of sphingolipids in yeast cell division. Mol. Microbiol. 84, 1018-1032.

Fang, M., Kearns, B. G., Gedvilaite, A., Kagiwada, S., Kearns, M., Fung, M. K. and Bankaitis, V. A. (1996). Kes1p shares homology with human oxysterol binding protein and participates in a novel regulatory pathway for yeast Golgiderived transport vesicle biogenesis. EMBO J. 15, 6447-6459.

Farhan, H. and Rabouille, C. (2011). Signalling to and from the secretory pathway. J. Cell Sci. 124, 171-180.

Funato, K. and Riezman, H. (2001). Vesicular and nonvesicular transport of ceramide from ER to the Golgi apparatus in yeast. J. Cell Biol. 155, 949-960.

Funato, K., Lombardi, R., Vallée, B. and Riezman, H. (2003). Lcb4p is a key regulator of ceramide synthesis from exogenous long chain sphingoid base in Saccharomyces cerevisiae. J. Biol. Chem. 278, 7325-7334.

Georgiev, A. G., Sullivan, D. P., Kersting, M. C., Dittman, J. S., Beh, C. T. and Menon, A. K. (2011). Osh proteins regulate membrane sterol organization but are not required for sterol movement between the ER and PM. Traffic 12, 1341-1355.

Gillon, A. D., Latham, C. F. and Miller, E. A. (2012). Vesicle-mediated ER export of proteins and lipids. Biochim. Biophys. Acta 1821, 1040-1049.

Haak, D., Gable, K., Beeler, T. and Dunn, T. (1997). Hydroxylation of Saccharomyces cerevisiae ceramides requires Sur2p and Scs7p. J. Biol. Chem. 272, 29704-29710.

Hanada, K., Kumagai, K., Yasuda, S., Miura, Y., Kawano, M., Fukasawa, M. and Nishijima, M. (2003). Molecular machinery for non-vesicular trafficking of ceramide. Nature 426, 803-809.

Horvath, A., Sütterlin, C., Manning-Krieg, U., Movva, N. R. and Riezman, H. (1994). Ceramide synthesis enhances transport of GPI-anchored proteins to the Golgi apparatus in yeast. EMBO J. 13, 3687-3695.

Im, Y. J., Raychaudhuri, S., Prinz, W. A. and Hurley, J. H. (2005). Structural mechanism for sterol sensing and transport by OSBP-related proteins. Nature 437, 154-158.

Johansen, J., Ramanathan, V. and Beh, C. T. (2012). Vesicle trafficking from a lipid perspective: Lipid regulation of exocytosis in Saccharomyces cerevisiae. Cell. Logist. 2, 151-160.

Kagiwada, S. and Hashimoto, M. (2007). The yeast VAP homolog Scs2p has a phosphoinositide-binding ability that is correlated with its activity. Biochem. Biophys. Res. Commun. 364, 870-876.

Kagiwada, S., Kearns, B. G., McGee, T. P., Fang, M., Hosaka, K. and Bankaitis, V. A. (1996). The yeast BSD2-1 mutation influences both the requirement for phosphatidylinositol transfer protein function and derepression of phospholipid biosynthetic gene expression in yeast. Genetics 143, 685-697.

Kagiwada, S., Hosaka, K., Murata, M., Nikawa, J. and Takatsuki, A. (1998). The Saccharomyces cerevisiae SCS2 gene product, a homolog of a synaptobrevinassociated protein, is an integral membrane protein of the endoplasmic reticulum and is required for inositol metabolism. J. Bacteriol. 180, 1700-1708.

Kajiwara, K., Watanabe, R., Pichler, H., Ihara, K., Murakami, S., Riezman, H. and Funato, K. (2008). Yeast ARV1 is required for efficient delivery of an early GPI intermediate to the first mannosyltransferase during GPI assembly and controls lipid flow from the endoplasmic reticulum. Mol. Biol. Cell 19, 2069-2082.

Kajiwara, K., Muneoka, T., Watanabe, Y., Karashima, T., Kitagaki, H. and Funato, K. (2012). Perturbation of sphingolipid metabolism induces endoplasmic reticulum stress-mediated mitochondrial apoptosis in budding yeast. Mol. Microbiol. 86, 1246-1261.

Kawano, M., Kumagai, K., Nishijima, M. and Hanada, K. (2006). Efficient trafficking of ceramide from the endoplasmic reticulum to the Golgi apparatus requires a VAMP-associated protein-interacting FFAT motif of CERT. J. Biol. Chem. 281, 30279-30288.

Kudo, N., Kumagai, K., Tomishige, N., Yamaji, T., Wakatsuki, S., Nishijima, M., Hanada, K. and Kato, R. (2008). Structural basis for specific lipid recognition by CERT responsible for nonvesicular trafficking of ceramide. Proc. Natl. Acad. Sci. USA 105, 488-493.

Kung, L. F., Pagant, S., Futai, E., D'Arcangelo, J. G., Buchanan, R., Dittmar, J. C., Reid, R. J., Rothstein, R., Hamamoto, S., Snapp, E. L. et al. (2012). Sec24p and Sec16p cooperate to regulate the GTP cycle of the COPII coat. EMBO J. 31, 1014-1027.

Lev, S. (2012). Nonvesicular lipid transfer from the endoplasmic reticulum. Cold Spring Harb. Perspect. Biol. 4, a013300.

Levine, T. P., Wiggins, C. A. and Munro, S. (2000). Inositol phosphorylceramide synthase is located in the Golgi apparatus of Saccharomyces cerevisiae. Mol. Biol. Cell 11, 2267-2281.

Loewen, C. J., Roy, A. and Levine, T. P. (2003). A conserved ER targeting motif in three families of lipid binding proteins and in Opi1p binds VAP. EMBO J. 22, 2025-2035

Lorente-Rodríguez, A. and Barlowe, C. (2011). Requirement for Golgi-localized $\mathrm{PI}(4) \mathrm{P}$ in fusion of COPII vesicles with Golgi compartments. Mol. Biol. Cell 22, 216-229.

Malathi, K., Higaki, K., Tinkelenberg, A. H., Balderes, D. A., AlmanzarParamio, D., Wilcox, L. J., Erdeniz, N., Redican, F., Padamsee, M., Liu, Y. 
et al. (2004). Mutagenesis of the putative sterol-sensing domain of yeast Niemann Pick C-related protein reveals a primordial role in subcellular sphingolipid distribution. J. Cell Biol. 164, 547-556.

Miller, E. A. and Barlowe, C. (2010). Regulation of coat assembly-sorting things out at the ER. Curr. Opin. Cell Biol. 22, 447-453.

Nagiec, M. M., Nagiec, E. E., Baltisberger, J. A., Wells, G. B., Lester, R. L. and Dickson, R. C. (1997). Sphingolipid synthesis as a target for antifungal drugs. Complementation of the inositol phosphorylceramide synthase defect in a mutant strain of Saccharomyces cerevisiae by the AUR1 gene. J. Biol. Chem. 272, 9809-9817.

Perry, R. J. and Ridgway, N. D. (2005). Molecular mechanisms and regulation of ceramide transport. Biochim. Biophys. Acta 1734, 220-234.

Perry, R. J. and Ridgway, N. D. (2006). Oxysterol-binding protein and vesicleassociated membrane protein-associated protein are required for sterol-dependent activation of the ceramide transport protein. Mol. Biol. Cell 17, 2604-2616.

Prinz, W. A. (2010). Lipid trafficking sans vesicles: where, why, how? Cell 143, 870-874.

Sambrook, J., Fritsch, E. F. and Maniatis, T. (1989). Molecular Cloning: A Laboratory Manual. Cold Spring Harbor, NY. Cold Spring Harbor Laboratory Press.

Sato, K. and Nakano, A. (2007). Mechanisms of COPII vesicle formation and protein sorting. FEBS Lett. 581, 2076-2082.

Sato, K., Sato, M. and Nakano, A. (2001). Rer1p, a retrieval receptor for endoplasmic reticulum membrane proteins, is dynamically localized to the Golgi apparatus by coatomer. J. Cell Biol. 152, 935-944.

Schönbächler, M., Horvath, A., Fassler, J. and Riezman, H. (1995). The yeast spt14 gene is homologous to the human PIG-A gene and is required for GPI anchor synthesis. EMBO J. 14, 1637-1645.
Schulz, T. A., Choi, M. G., Raychaudhuri, S., Mears, J. A., Ghirlando, R., Hinshaw, J. E. and Prinz, W. A. (2009). Lipid-regulated sterol transfer between closely apposed membranes by oxysterol-binding protein homologues. J. Cell Biol. 187, 889-903.

Shindiapina, P. and Barlowe, C. (2010). Requirements for transitional endoplasmic reticulum site structure and function in Saccharomyces cerevisiae. Mol. Biol. Cell 21, 1530-1545

Stefan, C. J., Manford, A. G., Baird, D., Yamada-Hanff, J., Mao, Y. and Emr, S. D. (2011). Osh proteins regulate phosphoinositide metabolism at ER-plasma membrane contact sites. Cell 144, 389-401.

Tomishige, N., Kumagai, K., Kusuda, J., Nishijima, M. and Hanada, K. (2009). Casein kinase Igamma2 down-regulates trafficking of ceramide in the synthesis of sphingomyelin. Mol. Biol. Cell 20, 348-357.

van Meer, G., Voelker, D. R. and Feigenson, G. W. (2008). Membrane lipids: where they are and how they behave. Nat. Rev. Mol. Cell Biol. 9, 112-124.

Vihervaara, T., Jansen, M., Uronen, R. L., Ohsaki, Y., Ikonen, E. and Olkkonen, V. M. (2011). Cytoplasmic oxysterol-binding proteins: sterol sensors or transporters? Chem. Phys. Lipids 164, 443-450.

Watanabe, R., Funato, K., Venkataraman, K., Futerman, A. H. and Riezman, H. (2002). Sphingolipids are required for the stable membrane association of glycosylphosphatidylinositol-anchored proteins in yeast. J. Biol. Chem. 277, 49538-49544.

Yano, T., Inukai, M. and Isono, F. (2004). Deletion of OSH3 gene confers resistance against ISP-1 in Saccharomyces cerevisiae. Biochem. Biophys. Res. Commun. 315, 228-234.

Yorimitsu, T. and Sato, K. (2012). Insights into structural and regulatory roles of Sec16 in COPII vesicle formation at ER exit sites. Mol. Biol. Cell 23, 2930-2942. 